\title{
DIFFERENT MORPHOLOGICAL STRUCTURES OF BREAST TUMORS DEMONSTRATE INDIVIDUAL DRUG RESISTANCE GENE EXPRESSION PROFILES
}

\author{
T.S. Gerashchenko ${ }^{1,2}$, E.V. Denisov ${ }^{1,2, *}$, N.M. Novikov ${ }^{1,3}$, L.A. Tashireva ${ }^{4}$, E.V. Kaigorodova ${ }^{4}$, \\ O.E. Savelieva, ${ }^{2,4}$ M.V. Zavyalova ${ }^{4}$, N.V. Cherdyntseva ${ }^{1,2}$, V.M. Perelmuter ${ }^{4}$ \\ ${ }^{1}$ Laboratory of Molecular Oncology and Immunology, Cancer Research Institute, \\ Tomsk National Research Medical Center, Tomsk 634050, Russia \\ ${ }^{2}$ Laboratory for Translational Cellular and Molecular Biomedicine, \\ Tomsk State University, Tomsk 634050, Russia \\ ${ }^{3}$ Department of Cytology and Genetics, Tomsk State University, Tomsk 634050, Russia \\ ${ }^{4}$ Department of General and Molecular Pathology, Cancer Research Institute, \\ Tomsk National Research Medical Center, Tomsk 634050, Russia
}

\begin{abstract}
Aim: To identify gene expression profiles involved in drug resistance of different morphological structures (tubular, alveolar, solid, trabecular, and discrete) presented in breast cancer. Material and Methods: Ten patients with luminal breast cancer have been included. A laser microdissection-assisted microarrays and qRT-PCR were used to perform whole-transcriptome profiling of different morphological structures, to select differentially expressed drug response genes, and to validate their expression. Results: We found 27 differentially expressed genes $(p<0.05)$ encoding drug uptake $(S L C 1 A 3, S L C 23 A 2$, etc.) and efflux $(A B C C 1, A B C G 1$, etc.) transporters, drug targets (TOP2A, TYMS, and Tubb3), and proteins that are involved in drug detoxification (NAT1 and $A L D H 1 B 1)$, cell cycle progression (CCND1, AKT1, etc.), apoptosis (CASP3, TXN2, etc.), and DNA repair (BRCA1 and USP11). Each type of structures showed an individual gene expression profile related to resistance and sensitivity to anticancer drugs. However, most of the genes $(19 / 27 ; p<0.05)$ were expressed in alveolar structures. Functional enrichment analysis showed that drug resistance is significantly associated with alveolar structures. Other structures demonstrated the similar number (10-13 out of 27) of expressed genes; however, the spectrum of resistance and sensitivity to different anticancer drugs varied. Conclusion: Different morphological structures of breast cancer show individual expression of drug resistance genes.
\end{abstract}

Key Words: breast cancer, tumor heterogeneity, gene expression, chemotherapy, drug resistance.

The problem of cancer heterogeneity constitutes a significant diagnostic and therapeutic challenge. Intratumor diversity implies the coexistence of different populations (subclones) with individual genetic alterations that affect their phenotype and biological behavior including, for example, ability to respond to chemotherapy.

Breast cancer (BC) represents an attractive model to study the influence of intratumor heterogeneity on chemotherapy response. BC comprises a highly heterogeneous group of diseases that is composed of different histological types with distinct morphologies and behaviors. The most widespread form of BC, invasive carcinoma no special type (IC NST), is represented by different architectural arrangements of tumor cells or morphological structures: tubular, alveolar, solid, trabecular, and discrete groups [1]. Such intratumor morphological heterogeneity is a common phenomenon in IC NST. Up to $80 \%$ of IC NST shows the presence of 3-5 different types of morphological structures [2].

$\mathrm{BC}$ treatment is well-known to be challenging due to multidrug resistance and corresponding drug inefficacy [3]. Our previous studies showed that neoadjuvant chemotherapy (NAC) efficiency of BC sig-

Submitted: May 18, 2018.

*Correspondence: E-mail: d_evgeniy@oncology.tomsk.ru Abbreviations used: BC - breast cancer; IC NST - invasive carcinoma no special type; NAC - neoadjuvant chemotherapy. nificantly depends on the intratumor morphological heterogeneity. A poor response to NAC is associated with the presence of alveolar, trabecular, or discrete groups in breast tumors, while the association was more significant in premenopausal patients. In contrast, a good response to chemotherapy was found in BCs with solid structures [2].

Molecular features of morphological structures that could explain their role in chemotherapy susceptibility are still unclear. Previously, we found that genes encoding $A B C$ transporters are differentially expressed between various morphological structures [4]. Our resent study showed that different morphological structures represent transcriptionally distinct populations of tumor cells with varying degrees of epithelialmesenchymal transition and stemness [5]. Based on these results, we assumed that various morphological structures carry specific characteristics which modify chemotherapy efficiency.

Thus, in this study, we aimed to assess gene expression features of different morphological structures in $\mathrm{BC}$ that contribute to their drug susceptibility and to describe a drug resistance/sensitivity profile of each structure.

\section{MATERIALS AND METHODS}

Patients and samples. Ten patients with IC NST of luminal subtypes (age range 40-65; mean age $53.00 \pm 9.28$ ) diagnosed and treated in the Cancer 
Research Institute of Tomsk NRMC (Tomsk, Russia) were enrolled in the study. All cases did not receive any preoperative therapy. Clinical and pathological characteristics are shown in Table 1.

Fresh samples of the breast tumor and adjacent normal tissues were obtained during surgery, placed in liquid nitrogen and stored at $-80^{\circ} \mathrm{C}$ until laser microdissection. The procedures followed in this study were in accordance with the Helsinki Declaration (1964, amended in 1975 and 1983). Ethical approval was obtained by the ethical committee of Cancer Research Institute, Tomsk NRMC. Approval reference is 10 (29 September 2011). All patients signed an informed consent for voluntary participation.

Laser microdissection and RNA isolation. All five types of morphological structures were observed in 7 out of 10 cases, whereas tubular structures were absent in the three patients (Table 1). Tubular, alveolar, solid, trabecular structures (50-120 samples of each structure), and discrete groups of tumor cells (300350 samples) were identified (Fig. 1, a) and isolated from $5 \mu \mathrm{m}$-thick hematoxylin and eosin stained sections of frozen tumor samples using PALM MicroBeam laser capture microdissection (Carl Zeiss, Germany). In addition, normal ducts (90-120 samples) were isolated from sections of normal breast tissue samples. In total, we obtained 47 microdissected samples of different structures ( 10 samples each of alveolar, solid, trabecular, and discrete groups and 7 samples of tubular structures) and 10 samples of normal ducts.

RNA was extracted from the microdissected samples by RNeasyPlus Micro Kit (Qiagen, USA) according to the manufacturer's instructions. RNA integrity number (RIN) was assessed using the 2200 TapeStation instrument and High Sensitivity RNA ScreenTape (Agilent, USA). Average RIN of samples was 5.6.

Microarray analysis. Three biological replicates of each type of morphological structures and normal breast epithelia (18 samples in total) were enrolled in gene expression profiling. In addition, each sample analyzed by gene expression microarrays was represented by tens and hundreds of structures of the same type (90-120 tubular, 90-120 alveolar, 90-120 trabecular, 50-60 solid, and 300-350 discrete groups). Other words, each sample contained many replicates of the same structures.

RNA samples of the microdissected samples were amplified using the Ovation PicoSLWTA System V2 kit (NuGEN, USA). Transcriptome profiling was performed using the SurePrint G3 Human GE v2, 8×60K microarrays (Agilent, USA), scanning - using a SureScan Microarray Scanner (Agilent, USA). All procedures were performed as previously described [5]. The microarray data is available in the Gene Expression Omnibus (GEO, GSE80754). The stored data were evaluated using the $\mathrm{R}$ software (R Development Core Team, 2008) and the limma package from Bioconductor [6]. Log mean spot signals were taken for further analysis. Expression levels were normalized to normal breast epithelia (ducts).

$\boldsymbol{q R T}$-PCR. RNA samples of the microdissected samples of 7 cases (39 samples in total; Table 1) were subjected to reverse transcription, ligation, and whole transcriptome amplification (QuantiTect Whole Transcriptome kit, Qiagen, USA). The amplified cDNA was used for qRT-PCR analysis of five genes (ABCG1, SLC25A13, SLC23A2, NAT1, and PIK3C3) to validate gene expression microarrays. qRT-PCR conditions, the temperature profile, and the algorithm for calculating expression levels are given in our previous study [7]. The results were presented as log2 foldchanges in the expression of the gene of interest relative to housekeeping genes (ACTB1 and GAPDH) and normal breast epithelia.

Statistical and functional enrichment analyses. A moderated t-statistic as implemented in the limma package was used to rank genes for differential expression between each type of morphological structures and normal breast epithelia. The Pearson correlation coefficient was used to assess the correlation between gene expression levels obtained by microarrays and QRT-PCR. Up- and down-regulated genes with $\mid$ log-fold-change $\mid \geqslant \log _{2} 1.5$ and an unadjusted $p$-value $<0.05$ were used in functional enrichment analysis by Qiagen's Ingenuity Pathway Analysis (IPA, Qiagen). The disease and functions analysis within IPA results was applied to find processes associated with drug resistance and the results were discussed using the following values: $-\log 10$ ( $p$-value), or a negative log of the $p$-value derived from the Fisher's Exact test.

\section{RESULTS AND DISCUSSION}

Based on the data of gene expression microarrays of different morphological structures, we estimated the expression of genes that are involved in the main mechanisms of drug susceptibility: drug uptake, efflux, and detoxification, the presence of drug targets, cell cycle arrest, apoptosis, and DNA repair.

Table 1. Clinicopathological characteristics of BC patients included in gene expression microarrays and qRT-PCR

\begin{tabular}{|c|c|c|c|c|c|c|c|c|c|c|}
\hline Cases & Age (yr) & Grade & TNM & Molecular subtype & ER & PR & HER2 & Ki-67, \% & Morphological structures & Analysis \\
\hline 1 & 46 & 2 & T2NOMO & Lum B HER2- & + & + & 0 & 34 & all structures & Gene expres- \\
\hline 2 & 40 & 2 & $\mathrm{~T} 2 \mathrm{~N} 2 \mathrm{M} 0$ & Lum B HER2- & + & + & $1+$ & 24 & all structures & sion micro- \\
\hline 3 & 49 & 2 & T2NOMO & Lum B HER2+ & + & + & $2+$ & 45 & all structures & array \\
\hline 4 & 59 & 2 & $\mathrm{~T} 2 \mathrm{~N} 3 \mathrm{M} 0$ & Lum B HER2+ & + & + & $2+$ & 38 & all structures & \\
\hline 5 & 49 & 2 & T1NOMO & Lum B HER2- & + & + & $1+$ & 37 & all structures & \\
\hline 6 & 65 & 2 & T1NOMO & Lum A & + & + & $1+$ & 19 & all structures & \\
\hline 7 & 42 & 3 & T1N1M0 & Lum B HER2- & + & + & $1+$ & 24 & alv, sol, trab, discr & qRT-PCR \\
\hline 8 & 53 & 1 & T1N0M0 & Lum B HER2- & + & - & 0 & 34 & alv, sol, trab, discr & \\
\hline 9 & 62 & 2 & T2NOMO & Lum B HER2- & + & - & 0 & 27 & all structures & \\
\hline 10 & 65 & 1 & T2NOMO & Lum B HER2- & + & + & $1+$ & 25 & alv, sol, trab, discr & \\
\hline
\end{tabular}

Note: TNM - tumor-node-metastasis classification; Lum - luminal; yr - years; ER - estrogen receptors; PR - progesterone receptors; "+" - presence; "-" absence; tub - tubular; alv - alveolar; sol - solid; trab - trabecular; discr - discrete. HER2+ status was complemented by FISH analysis. 
First, we assessed the expression of genes ( $A B C B 1$, GSTP1, CYP, BAX, etc.) that were described to play a critical role in tumor drug resistance/sensitivity. Surprisingly, no significant differential expression of these genes was observed between various structures. Some genes were absent in morphological structures whereas others were not significantly underor overexpressed. However, there were exceptions such as ABCG2 and GSTP1 genes whose expression was considerably down-regulated in all structures excluding discrete groups of tumor cells. These structures also showed the significant up-regulation of SCL1A1 and SLC7A2 genes. In addition, the APC gene was substantially overexpressed only in trabecular structures (Table 2).

Second, given the above-mentioned results, we performed an additional analysis of the gene expression microarrays and found other genes that also contribute to susceptibility to anticancer drugs according to the literature data (Table 3). In Fig. 1, b, we summarized genes whose expression was significantly differential between different morphological structures. Most of the drug response genes (19 out of 27) were found to be considerably expressed in alveolar structures, whereas other morphological structures demonstrated almost similar number of expressed genes. In particular, 13 genes were significantly expressed in solid and trabecular structures and 10 - in tubular and discrete groups of tumor cells (Fig. 1, b).

Functional enrichment analysis of under- and overexpressed genes (the expressional level $>1.5$ or -1.5 , $p<0.05)$ in morphological structures also showed an increased drug resistance profile of alveolar structures. In particular, the processes such as "drug resistance of cells" and "chemotherapy resistance of carcinoma cell lines" were significantly associ-
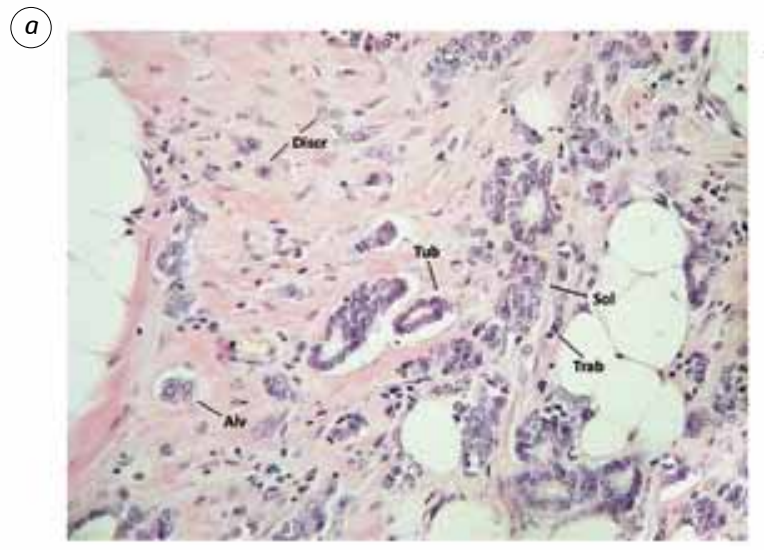

(b)

\begin{tabular}{|c|c|c|c|c|c|c|}
\hline & Genes & Tub & Alv & Sed & Trab & Deser \\
\hline \multirow{4}{*}{ Drug uptake } & $S: C I A 3$ & .1 .79 & .213 & .198 & $5.28^{\circ}$ & $.3 .94^{\circ}$ \\
\hline & $\sec 23,42$ & 0.63 & -106 & 0.54 & $-251^{\circ}$ & $.250^{\circ}$ \\
\hline & SICZSAI3 & 0.58 & $283^{\circ}$ & 0.38 & $309^{\circ}$ & .100 \\
\hline & $S 202042$ & NO & -120 & $4.71^{\circ}$ & -1.03 & -1.41 \\
\hline \multirow{4}{*}{ Drug ethax } & ABCA12 & $499^{\circ}$ & $5.53^{\circ}$ & 5.17 & $500^{\circ}$ & $3.70^{\circ}$ \\
\hline & ABCCI & 240 & $1.32^{\circ}$ & 0.8 & 2.84 & 201 \\
\hline & Ascci1 & $500^{+}$ & $4.16^{\circ}$ & $580^{\circ}$ & $270^{\circ}$ & $3.70^{*}$ \\
\hline & ASCES & 203 & $3.30^{\circ}$ & 2.60 & 2.18 & No \\
\hline \multirow{2}{*}{$\begin{array}{l}\text { Drug } \\
\text { decouctisation }\end{array}$} & NATI & $500^{\circ}$ & $5 . \pi^{\circ}$ & $550^{\circ}$ & $565^{\circ}$ & $3.40^{*}$ \\
\hline & Atoritas & 223 & $335^{\circ}$ & ND & 1.38 & 2.74 \\
\hline \multirow{3}{*}{$\begin{array}{l}\text { The presence } \\
\text { of drue targets }\end{array}$} & TOP2A & 286 & $3,4^{*}$ & $3.75^{\circ}$ & $400^{\circ}$ & 2.02 \\
\hline & mes & $350^{*}$ & $167^{\circ}$ & 3200 & $20^{\circ}$ & 2.51 \\
\hline & notos & xes: & $1.44^{\circ}$ & $29 \pi$ & $510^{\circ}$ & $360^{\circ}$ \\
\hline \multirow{6}{*}{$\begin{array}{l}\text { Cell cycle } \\
\text { arrest }\end{array}$} & CONS20 & $451^{\circ}$ & 3.48* & 2.21 & $4000^{\circ}$ & $320^{\circ}$ \\
\hline & CONDI & $242^{*}$ & 189 & $201^{*}$ & $1.7 n$ & 0.49 \\
\hline & PXK3C3 & 295 & $3.70^{\circ}$ & 2.99 & $3.12^{*}$ & 2.45 \\
\hline & AKTI & $3.63^{\circ}$ & $3.75^{\circ}$ & 2.98 & 3.14 & 1.91 \\
\hline & FUKt & 2.21 & $283^{\circ}$ & $283^{\circ}$ & 2.47 & $3.36^{\circ}$ \\
\hline & UลEZS & $243^{*}$ & $2.977^{*}$ & 2.10 & 204 & 1.58 \\
\hline \multirow{6}{*}{ Apoptosis } & 2002 & 173 & क.s & 1.18 & $2.87^{\circ}$ & 2.14 \\
\hline & $x=0$ is & 161 & 100 & $2 \mathrm{~g} \mathrm{~F}^{\circ}$ & 0.90 & 2.35 \\
\hline & $\min 31$ & NO & $2.11^{*}$ & $2.2^{\circ}$ & $-2.29^{*}$ & $2.188^{\circ}$ \\
\hline & CASPB & 0.95 & 160 & $20 \%$ & 2.02 & 0.58 \\
\hline & อะ & 1.36 & $4.25^{\circ}$ & 1.14 & 1.47 & 2.09 \\
\hline & use 15 & 2.65 & $3.16^{\circ}$ & 2.60 & NO & 236 \\
\hline \multirow{2}{*}{ CNA repsir } & BACA1 & $285^{\circ}$ & 1.36 & $5.82^{\circ}$ & ND & 10 \\
\hline & USP11 & 0.31 & $105^{\circ}$ & 2.03 & 1.13 & $3.55^{\circ}$ \\
\hline
\end{tabular}
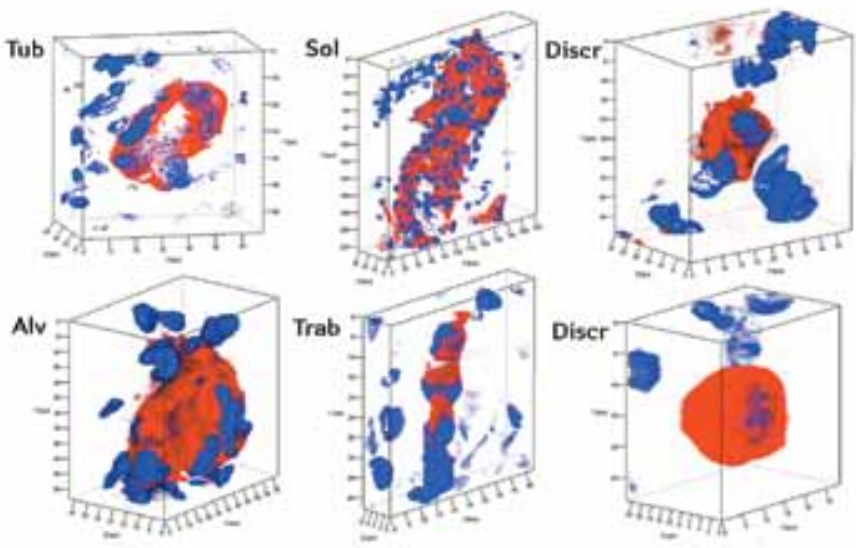

(c)

\begin{tabular}{|c|c|c|c|c|c|c|}
\hline & & Tub & ave & sol & Trab & Dover \\
\hline \multirow{2}{*}{ Sthoroureat } & Semitivaty & & & & & \\
\hline & Rowing & & & & & \\
\hline \multirow{2}{*}{ Tasures } & Senditidy & & & & & \\
\hline & hasiones & & & & & \\
\hline \multirow{2}{*}{ Anthacyulion: } & Senitivaty & & & & & \\
\hline & Frimane & & & & & \\
\hline \multirow{2}{*}{ Mertotremate } & Serouthay & & & & & \\
\hline & Kenitres: & & & & & \\
\hline \multirow{2}{*}{ Complotiencin } & Senitivaty & & & & & \\
\hline & Renitung & & & & & \\
\hline \multirow{2}{*}{ Hercopten } & Senitiony & & & & & \\
\hline & Foliting & & & & & \\
\hline \multirow{2}{*}{ Crithphoupharicte } & Senditiaty & & & & & \\
\hline & Resionie & & & & & \\
\hline \multirow{2}{*}{ Eoposide } & Semitivety & & & & & \\
\hline & Knomern & & & & & \\
\hline \multirow{2}{*}{ Matinum trues } & Senitiaty & & & & & \\
\hline & Renstame & & & & & \\
\hline \multirow{2}{*}{ Tamsalten } & Sentitiay & & & & & \\
\hline & Aniens & & & & & \\
\hline \multirow{2}{*}{$\begin{array}{l}\text { Gencitatine, } \\
\text { Sidurntine }\end{array}$} & Senitiay & & & & & \\
\hline & Mesiazuz & & & & & \\
\hline & & & & & 1 & \\
\hline & & & & & 2 & \\
\hline & & & & & , & \\
\hline & & & & & 4 & \\
\hline
\end{tabular}

Fig. 1. Expression levels of drug resistance and sensitivity genes in different morphological structures. (a) Hematoxylin and eosin stained section of the breast tumor with different morphological structures (left): tubular (Tub), alveolar (Alv), solid (Sol), trabecular (Trab), and discrete (Discr) and their 3D immunofluorescence images (right). Tubular structures are represented by the tubeshaped aggregations of tumor cells. Alveolar structures have a rounded (spheroidal) shape and contain up to 30 tumor cells. Solid structures are represented by the large shapeless groups of tens and hundreds of tumor cells. Trabecular structures are formed by one or two rows of tumor cells. Discrete groups of tumor cells are defined by arrangements of $2-5$ cells and/or single cells. Red color indicates the cytoplasmic expression of cytokeratin 7, blue - DAPI staining (nucleus) [5]. (b) Expression levels of drug resistance and sensitivity genes in different morphological structures. ND - not determined; *expression level at $p<0.05$. The color intensity is proportional to gene expression levels from low (green) to high (red). (c) The panel of resistance and sensitivity of different morphological structures of IC NST to anticancer drugs. The scales (1-3 and 1-4) show the number of overexpressed genes associated with sensitivity and resistance to any drug. Anthracyclines (doxorubicin and epirubicin), taxanes (paclitaxel), and platinum drugs (cisplatin) 
Table 2. Expression levels of drug resistance and sensitivity genes in different morphological structures of $B C$

\begin{tabular}{|c|c|c|c|c|c|c|}
\hline Drug resistance mechanisms & Genes & Tub & Alv & Sol & Trab & Discr \\
\hline \multirow{5}{*}{ Drug uptake } & SLC19A1 & ND & 0.85 & 0.48 & 0.36 & 0.45 \\
\hline & SLC29A1 & -0.52 & -1.47 & -1.42 & -2.37 & -0.35 \\
\hline & SLC1A1 & $3.78^{\star}$ & $3.18^{\star}$ & $3.59^{\star}$ & $3.48^{*}$ & 2.6 \\
\hline & SLC7A2 & $3.06^{\star}$ & $3.16^{\star}$ & $4.11^{*}$ & $3.08^{*}$ & 1.57 \\
\hline & SLC7A11 & 1.7 & $3.29 *$ & $3.32^{\star}$ & 1.83 & $3.21^{\star}$ \\
\hline \multirow{6}{*}{ Drug efflux } & $A B C B 1$ & 0.38 & -0.61 & ND & 1.27 & -0.68 \\
\hline & $A B C B 4$ & -0.68 & -0.38 & -1.11 & -1.4 & ND \\
\hline & ABCB5 & 0.34 & ND & -0.27 & 0.71 & -0.27 \\
\hline & $A B C C 3$ & -0.50 & 1.38 & 1.29 & 1.51 & 1.04 \\
\hline & $A B C C 5$ & 1.62 & 1.24 & 1.37 & 1.91 & 1.84 \\
\hline & $A B C G 2$ & $-2.8^{*}$ & $-2.7^{\star}$ & $-2.1^{\star}$ & $-2.38^{\star}$ & -1.33 \\
\hline \multirow{7}{*}{ Drug detoxification } & $D P Y D$ & -0.70 & -0.97 & -1.1 & -0.4 & -0.44 \\
\hline & CYP3A5 & -0.53 & -1.02 & ND & ND & -0.64 \\
\hline & CYP2D6 & 0.24 & 0.5 & 0.37 & 1.07 & 0.29 \\
\hline & CYP2C8 & ND & -0.33 & 0.62 & 0.62 & -0.33 \\
\hline & GSTP1 & $-2.96^{\star}$ & $-3.39^{*}$ & $-2.1^{*}$ & $-2.6^{\star}$ & -0.4 \\
\hline & CYP1A & -0.56 & 0.57 & 0.32 & 1.6 & -0.32 \\
\hline & СYP2C9 & -0.53 & -0.46 & -0.42 & -0.38 & ND \\
\hline \multirow[t]{2}{*}{ The presence of drug targets } & TOP1 & 0.85 & $\begin{array}{l}\mathrm{ND} \\
\end{array}$ & 0.48 & -0.52 & ND \\
\hline & CCNE1 & -2.32 & -1.23 & -1.99 & -1.30 & 0.95 \\
\hline \multirow{4}{*}{ Cell cycle arrest } & CDK2 & 1.64 & ND & ND & 0.4 & 0.57 \\
\hline & CDK4 & 0.32 & 0.7 & 0.82 & 0.41 & ND \\
\hline & CDKN1A & ND & ND & -0.55 & -0.65 & ND \\
\hline & CDKN2A & 2.11 & 0.96 & 1.81 & 0.67 & 2.23 \\
\hline \multirow{6}{*}{ Apoptosis } & $B A D$ & 2.2 & 1.52 & 1.78 & -0.97 & 0.45 \\
\hline & TP53 & ND & -0.96 & -0.83 & -1.46 & ND \\
\hline & $B A X$ & -0.32 & ND & -0.46 & -1.78 & 0.34 \\
\hline & $A P C$ & 0.79 & ND & 2.4 & $3.11^{*}$ & 0.69 \\
\hline & CFLAR & 0.13 & 0.25 & 0.19 & 0.19 & 0.43 \\
\hline & $B c / 2$ & ND & 0.62 & 1.14 & -0.50 & 1.74 \\
\hline \multirow{7}{*}{ DNA repair } & BRCA2 & -0.64 & 0.26 & -0.24 & -0.57 & -0.43 \\
\hline & ERCC1 & -0.97 & -1.22 & -1.56 & -1.06 & -1.95 \\
\hline & ERCC3 & -0.56 & -2.18 & -1.38 & -0.77 & ND \\
\hline & ATM & 0.98 & ND & 0.29 & -0.36 & 1.41 \\
\hline & $X P A$ & -0.86 & 0.77 & ND & 2.54 & -1.68 \\
\hline & $X P C$ & $-2.71^{*}$ & $-2.22^{\star}$ & $-1.9^{\star}$ & -1.34 & -0.39 \\
\hline & MSH2 & 0.84 & ND & 1.02 & 0.44 & -1.90 \\
\hline
\end{tabular}

Note: Tub - tubular; Alv - alveolar; Sol - solid; Trab - trabecular; Discr - discrete; ND - not determined; ${ }^{*}$ expression level at $p<0.05$.

Table 3. Differentially expressed drug resistance and sensitivity genes in different morphological structures of IC NST

\begin{tabular}{|c|c|c|c|c|c|}
\hline Drug resistance mechanisms & Gene symbol & Full gene name & $\mathrm{R} / \mathrm{S}^{*}$ & Drugs affected & Ref. \\
\hline \multirow[t]{4}{*}{ Drug uptake } & SLC1A3 & Solute Carrier Family 1 Member 3 & $\mathrm{R}$ & cisplatin & {$[8]$} \\
\hline & SLC23A2 & Solute Carrier Family 23 Member 2 & $\mathrm{R}$ & $5 \mathrm{FU}$ & {$[9,10]$} \\
\hline & SLC25A13 & Solute Carrier Family 25 Member 13 & $\mathrm{~S}$ & camptothecin & [9] \\
\hline & SLC29A2 & Solute Carrier Family 29 Member 2 & $\mathrm{R}$ & gemcitabine, fludarabine & [11] \\
\hline \multirow[t]{4}{*}{ Drug efflux } & \multirow{2}{*}{$\begin{array}{c}A B C A 12 \\
A B C C 1\end{array}$} & \multirow{2}{*}{$\begin{array}{l}\text { ATP Binding Cassette Subfamily A Member } 12 \\
\text { ATP Binding Cassette Subfamily C Member } 1\end{array}$} & $\mathrm{R}$ & $5 \mathrm{FU}$, paclitaxel & \multirow{2}{*}{$\begin{array}{c}{[12]} \\
{[12,13]}\end{array}$} \\
\hline & & & $\mathrm{R}$ & $\begin{array}{c}\text { etoposide, CPA, } \\
\text { doxorubicin methotrexate }\end{array}$ & \\
\hline & $A B C C 11$ & ATP Binding Cassette Subfamily C Member 11 & $\mathrm{R}$ & $5 \mathrm{FU}$ & {$[12,14]$} \\
\hline & ABCG1 & ATP Binding Cassette Subfamily G Member 1 & $\overline{\mathrm{R}}$ & doxorubicin & {$[12]$} \\
\hline \multirow[t]{2}{*}{ Drug detoxification } & NAT1 & $\mathrm{N}$-Acetyltransferase 1 & $\mathrm{R}$ & etoposide & \multirow{2}{*}{$\begin{array}{c}{[15]} \\
{[16,17]}\end{array}$} \\
\hline & ALDH1B1 & Aldehyde Dehydrogenase 1 Family Member B1 & $\mathrm{R}$ & CPA & \\
\hline \multirow[t]{3}{*}{ The presence of drug targets } & TOP2A & DNA Topoisomerase II Alpha & $\mathrm{S}$ & anthracyclines & \multirow{3}{*}{$\begin{array}{l}{[18]} \\
{[19]} \\
{[20]}\end{array}$} \\
\hline & TYMS & Thymidylate Synthetase & $\mathrm{S}$ & $5 \mathrm{FU}$ & \\
\hline & Tubb3 & Tubulin Beta 3 Class III & $\mathrm{S}$ & taxanes & \\
\hline \multirow[t]{6}{*}{ Cell cycle arrest } & CDKN2D & Cyclin Dependent Kinase Inhibitor 2D & $\bar{R}$ & cisplatin & \multirow{6}{*}{$\begin{array}{c}{[21]} \\
{[22]} \\
{[23,24]} \\
{[25]} \\
{[26]} \\
{[27]}\end{array}$} \\
\hline & CCND1 & Cyclin D1 & $\mathrm{R}$ & tamoxifen & \\
\hline & \multirow{4}{*}{$\begin{array}{l}\text { PIK3C3 } \\
\text { AKT1 } \\
\text { PLK1 } \\
\text { UBE2S }\end{array}$} & \multirow{4}{*}{$\begin{array}{l}\text { Phosphatidylinositol 3-Kinase Catalytic Subunit Type } 3 \\
\text { AKT Serine/Threonine Kinase } 1 \\
\text { Polo Like Kinase } 1\end{array}$} & $\bar{R}$ & tamoxifen & \\
\hline & & & $\mathrm{R}$ & cisplatin & \\
\hline & & & $\mathrm{R}$ & trastuzumab, paclitaxel & \\
\hline & & & $\mathrm{S}$ & taxanes & \\
\hline \multirow[t]{6}{*}{ Apoptosis } & $T X N 2$ & Thioredoxin 2 & $\mathrm{~S}$ & tamoxifen & \multirow{4}{*}{$\begin{array}{l}{[28]} \\
{[29]} \\
{[30]} \\
{[31]}\end{array}$} \\
\hline & XP01 & Exportin 1 & $\overline{\mathrm{R}}$ & doxorubicin, etoposide & \\
\hline & \multirow{2}{*}{$\begin{array}{l}\text { mir-31 } \\
\text { CASP3 }\end{array}$} & \multirow{2}{*}{$\begin{array}{l}\text { MicroRNA } 31 \\
\text { Caspase } 3\end{array}$} & $\mathrm{R}$ & paclitaxel & \\
\hline & & & $\mathrm{S}$ & $\begin{array}{l}\text { cisplatin, doxorubicin, } \\
\text { etoposide }\end{array}$ & \\
\hline & $B R I 3 B P$ & BRI3 Binding Protein & $\mathrm{R}$ & etoposide & \multirow{2}{*}{$\begin{array}{l}{[32]} \\
{[33]}\end{array}$} \\
\hline & USP15 & Ubiquitin Specific Peptidase 15 & $\mathrm{~S}$ & paclitaxel & \\
\hline \multirow[t]{3}{*}{ DNA repair } & BRCA1 & BRCA1, DNA repair associated & $\mathrm{S}$ & paclitaxel & [34] \\
\hline & \multirow[b]{2}{*}{ USP11 } & \multirow[b]{2}{*}{ Ubiquitin Specific Peptidase 11} & $\mathrm{R}$ & cisplatin, etoposide & \multirow[b]{2}{*}{ [35] } \\
\hline & & & $\mathrm{R}$ & $\begin{array}{l}\text { paclitaxel, doxorubicin, } \\
\text { epirubicin }\end{array}$ & \\
\hline
\end{tabular}

Note: $\mathrm{R}$ - resistance; $\mathrm{S}$ - sensitivity; 5FU - 5-fluorouracil; CPA - cyclophosphamide; Ref. - references; *in gene overexpression.

ated only with alveolar structures $(-\log 10(p$-value $)=$ 3.16 and 3.33, respectively; data not shown).

Finally, we validated the expression levels of five drug response genes (ABCG1, SLC25A13, SLC23A2, NAT1, and PIK3C3) yielded by microarrays using quantitative real-time polymerase chain reaction (qRT-PCR). The gene expression was positively correlated between microarrays and PCR ( $r=0.73 ; p<0.05$, Fig. 2). 


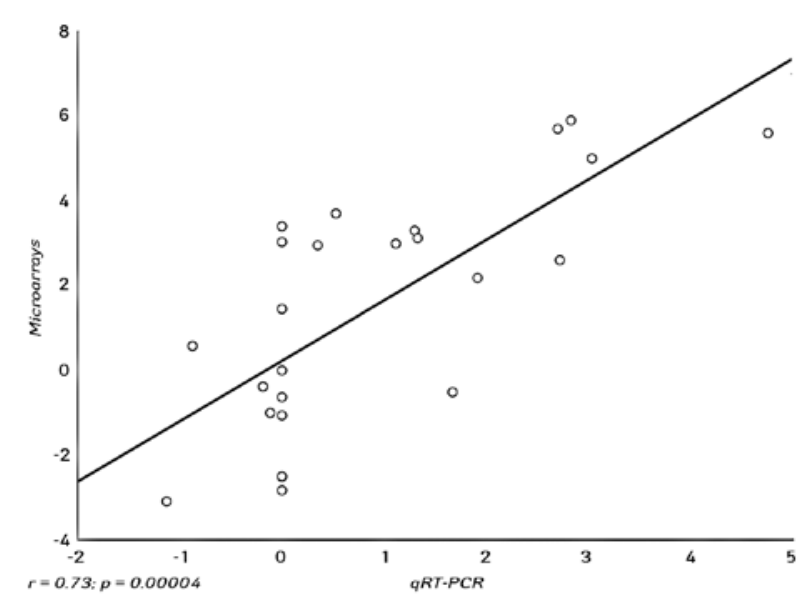

Fig. 2. Correlation between gene expression microarrays and qRT-PCR. Plot shows the log mean spot signals and the logtransformed expression levels of 5 genes detected by gene expression microarrays (y-axis) and qRT-PCR (x-axis), respectively

In general, the microarray data showed that all structures display common gene expression features. This includes increased drug detoxification through overexpression of the NAT1 gene and drug efflux through the $A B C A 12$ and $A B C C 11$ genes as well as taxane sensitivity through the high expression of the Tubb3 gene. Nevertheless, each morphological structure had specific drug susceptibility features. The expression of genes involved in the inhibition of druginduced apoptosis was frequently observed in alveolar and solid structures. Alveolar structures also showed upregulation of cell cycle genes, whereas trabecular structures more often displayed overexpression of drug uptake genes.

Despite the fact that most drug insensitivity genes were expressed in alveolar structures, total tumor chemoresistance is probably formed by the contribution of each type of morphological structures. Below, we summarized information about drug resistance and sensitivity features of different morphological structures in IC NST.

Tubular structures showed the significant overexpression of 10 genes involved in resistance to 5 -fluorouracil, capecitabine, etoposide, cisplatin, and tamoxifen (Fig. 1, b, c). Despite the afore-mentioned resistance, these structures displayed sensitivity to 5-fluorouracil and capecitabine as well as taxanes. It is of note that tubular structures were not characterized by expression of genes associated with resistance to anthracyclines, cyclophosphamide and others (Fig. 1, $c$ ) that makes these agents appropriate to be prescribed for the therapy of BCs composed of these morphological variants.

Alveolar structures were characterized by the significant expression of the broadest panel of 19 genes that confer resistance to almost all therapeutic agents used in the treatment of BC patients (Fig. 1, $b, c$ ). For example, only alveolar structures showed simultaneous expression of four $A B C$ transporter genes: $A B C G 1, A B C C 1, A B C A 12$ and $A B C C 11$ (Fig. 1, b), which efflux 5-fluorouracil, taxanes, anthracyclines, methotrexate, cyclophosphamide, and etoposide [12-14]. This probably explains the association of these structures with a lack of clinical response to NAC $[2,4,36]$. Given these data, the choice of an effective strategy to treat BCs containing alveolar structures is challenging. Nevertheless, these structures may be susceptible to camptothecin due to underexpression of the SLC25A13 which is unable to transfer this drug into mitochondria for its metabolism [9, 37] as well as to tamoxifen despite the PIK3C3 overexpression. Previous results suggested the involvement of the PIK3C3 overexpression in tamoxifen and anthracycline resistance through activation of autophagy [23, 24]; however, further studies are needed to confirm this.

Solid structures demonstrated the significant expression of 13 genes involved in chemotherapy response (Fig. 1, b). The distinctive feature of solid structures was the expression of 5 key genes: TYMS, Tubb3, BRCA1, TOP2A, and CASP3 that provide susceptibility to a broad spectrum of drugs (Fig. 1, C). In addition, the CASP3 gene associated with druginduced apoptosis was considerably overexpressed only in solid structures (Fig. 1, b). These observations together with the data provided in Fig. 1, $c$ indicate that solid structures possess resistance to trastuzumab, tamoxifen, and gemcitabine as well as simultaneous insensitivity and susceptibility to 5-fluorouracil, taxanes, anthracyclines, etoposide, and platinum-based drugs (Fig. 1, c). Thus, one may assume that solid structures are rather chemosensitive than drug-resistant. This suggestion is also supported by the fact that the presence of solid structures in breast tumors of premenopausal patients is associated with a good response to chemotherapy [2].

Trabecular structures showed the considerable expression of 13 genes that modulate drug response (Fig. 1, b). The distinctive feature of these structures was the significant underexpression of 3 of 4 SLC transporters: SLC25A13, SLC23A2, and SLC1A3 that are responsible for uptake of camptothecin, 5-fluorouracil, and platinum-based drugs [8-10]. The TXN2 gene implicated in tamoxifen resistance [28] was strongly overexpressed only in trabecular structures (Table 3, Fig. 1, b). In addition, DNA repair genes were either absent (BRCA1) or not considerably upregulated (USP11) in these morphological structures (Fig. 1,b). Probably, these expression features, namely a decreased work of SLC transporters, as well as other characteristics (Fig. 1, $b, c$ ), may explain the previous results about the association of trabecular structures with a poor response to NAC [2, 4, 36]. Nevertheless, it seems that trabecular structures can be sensitive to anthracyclines (Fig. 1, $C$ ) because of overexpression of the TOP2A gene as well as no well-defined role of the PIK3C3 gene in resistance to this drug as indicated above. In addition, SLC25A13 underexpression may be a marker for camptothecin sensitivity (Fig. 1, C). Interestingly, we did not find high expression of the $A B C B 1$ gene in trabecular structures as found in our previous study [4]. However, earlier work [4] did not 
apply statistical processing to find if the $A B C B 1$ gene was significantly under- or overexpressed in trabecular structures compared to normal breast epithelia. Moreover, the previous study [4] enrolled patients with luminal A BC.

Discrete groups of cells were characterized by the significant expression of 10 genes involved in chemotherapy response (Fig. 1, b, c). The distinctive feature of these structures was a low activity of drug sensitivity genes (Fig. 1, b). In particular, discrete groups showed the considerable overexpression of only Tubb3 gene which is a clinical marker of sensitivity to taxanes [20]. The recent study described discrete groups of tumor cells as associated with low NAC effectiveness in premenopausal patients [2]. Most likely, it can be related to the prevalence of drug resistance genes including $A B C C 11, A B C A 12, S L C 23 A 2$, and etc. and almost complete absence of chemosensitivity markers (Fig. 1, b, c). In addition, the highly heterogeneous composition of these morphological structures [5] may contribute to drug resistance and should be considered when an appropriate chemotherapy regimen is chosen.

It should be noted that drug resistance and sensitivity of different morphological structures mentioned above has a couple of drawbacks. First, the data discussed here concern so-called intrinsic drug resistance (that is presented before treatment) because $B C$ cases included in the gene expression profiling were NAC-naïve. Second, only genes which significantly under- and overexpressed $(p<0.05)$ in morphological structures compared to normal breast epithelia were used to make the drug resistance and sensitivity panel (Fig. 1, c). Nevertheless, other genes modulating drug response were also expressed in various morphological structures (Table 2), but differences were not significant. Third, the role of some genes provided in Table 3 and used for the construction of the drug susceptibility profile is not well-defined and further investigations are required.

\section{CONCLUSION}

Various morphological structures of IC NST previously described as transcriptionally distinct populations of tumor cells and to be associated with the chemotherapy efficacy differ amongst themselves in the expression of drug resistance and sensitivity genes. Simply stated, different morphological structures show individual drug susceptibility profiles. The most drugresistant phenotype is typical for alveolar structures which were earlier found to be implicated in NAC inefficiency $[2,4,36]$, whereas solid structures are more drug sensitive and were previously associated with a good response to chemotherapy [2]. In addition, each type of structures shows resistance and sensitivity to certain therapeutic agents that are associated with expression of specific genes. For example, alveolar structures may be susceptible to camptothecin and tamoxifen but are tolerant to 5-fluorouracil, taxanes and etc. Trabecular structures probably show potential sensitivity to camptothecin and anthracyclines and resistance to other drugs. Overall, different morphological structures contribute to the intrinsic drug resistance of BC and their assessment represent a simple method of predicting response to chemotherapy and an additional criterion for the selection of adequate therapy regimens.

\section{ACKNOWLEDGMENTS}

The study was supported by the Russian Science Foundation (grant \# 14-15-00318).

\section{REFERENCES}

1. Zavyalova MV, Perelmuter VM, Vtorushin SV, et al. The presence of alveolar structures in invasive ductal NOS breast carcinoma is associated with lymph node metastasis. Diagn Cytopathol 2013; 41: 279-82.

2. Gerashchenko TS, Zavyalova MV, Denisov EV, et al. Intratumoral morphological heterogeneity in breast cancer as a reflection of metastatic potential and tumor chemosensitivity. Acta Naturae 2017; 9: 56-67.

3. Martin HL, Smith L, Tomlinson DC. Multidrugresistant breast cancer: current perspectives. Breast Cancer (Dove Med Press) 2014; 6: 1-13.

4. Denisov EV, Litviakov NV, Zavyalova MV, et al. Intratumoral morphological heterogeneity of breast cancer: neoadjuvant chemotherapy efficiency and multidrug resistance gene expression. Sci Rep 2014; 4: 4709.

5. Denisov EV, Skryabin NA, Gerashchenko TS, et al. Clinically relevant morphological structures in breast cancer represent transcriptionally distinct tumor cell populations with varied degrees of epithelial-mesenchymal transition and CD44+CD24- stemness. Oncotarget 2017; 8: 61163-80.

6. Gentleman RC, Carey VJ, Bates DM, et al. Bioconductor: open software development for computational biology and bioinformatics. Genome Biol 2004; 5: R80.

7. Denisov EV, Gerashchenko TS, Zavyalova MV, et al. Invasive and drug resistant expression profile of different morphological structures of breast tumors. Neoplasma 2015; 62: $405-11$.

8. Konkimalla VB, Kaina B, Efferth T. Role of transporter genes in cisplatin resistance. In Vivo 2008; 22: 279-83.

9. Huang Y, Anderle P, Bussey KJ, et al. Membrane transporters and channels: role of the transportome in cancer chemosensitivity and chemoresistance. Cancer Res 2004; 64: 4294-301.

10. Januchowski R, Zawierucha P, Rucinski M, et al. Drug transporter expression profiling in chemoresistant variants of the A2780 ovarian cancer cell line. Biomed Pharmacother 2014; 68: 447-53.

11. Marin JJ, Briz O, Monte MJ, et al. Genetic variants in genes involved in mechanisms of chemoresistance to anticancer drugs. Curr Cancer Drug Targets 2012; 12: 402-38.

12. Zhang JT. Use of arrays to investigate the contribution of ATP-binding cassette transporters to drug resistance in cancer chemotherapy and prediction of chemosensitivity. Cell Res 2007; 17: 311-23.

13. Munoz M, Henderson $M$, Haber M, et al. Role of the MRP1/ABCC1 multidrug transporter protein in cancer. IUBMB Life 2007; 59: 752-7.

14. Guo Y, Kotova E, Chen ZS, et al. MRP8, ATP-binding cassette $\mathrm{C} 11$ (ABCC11), is a cyclic nucleotide efflux pump and a resistance factor for fluoropyrimidines 2 ', 3 '-dideoxycytidine and 9'-(2'-phosphonylmethoxyethyl)adenine. J Biol Chem 2003; 278: 29509-14. 
15. Rodrigues-Lima F, Dairou J, Busi F, et al. Human arylamine $\mathrm{N}$-acetyltransferase 1: a drug-metabolizing enzyme and a drug target? Curr Drug Targets 2010; 11: 759-66.

16. Bacolod MD, Lin SM, Johnson SP, et al. The gene expression profiles of medulloblastoma cell lines resistant to preactivated cyclophosphamide. Curr Cancer Drug Targets 2008; 8: 172-9.

17. Moreb JS, Mona M, Chang L-J, et al. Abstract 3762: The role of aldehyde dehydrogenase isoenzymes in cancer cell proliferation, migration and drug resistance. Cancer Res 2013; 73: 3762.

18. Di Leo A, Desmedt C, Bartlett JM, et al. HER2 and TOP2A as predictive markers for anthracycline-containing chemotherapy regimens as adjuvant treatment of breast cancer: a meta-analysis of individual patient data. Lancet Oncol 2011; 12: 1134-42.

19. Zhang N, Yin Y, Xu SJ, et al. 5-Fluorouracil: mechanisms of resistance and reversal strategies. Molecules 2008; 13: $1551-69$.

20. Kavallaris M. Microtubules and resistance to tubulinbinding agents. Nat Rev Cancer 2010;10: 194-204.

21. Arora S, Bisanz KM, Peralta LA, et al. RNAi screening of the kinome identifies modulators of cisplatin response in ovarian cancer cells. Gynecol Oncol 2010; 118: 220-7.

22. Roy PG, Thompson AM. Cyclin D1 and breast cancer. Breast 2006; 15: 718-27.

23. Backer JM. The regulation and function of Class III PI3Ks: novel roles for Vps34. Biochem J 2008; 410: 1-17.

24. Cook KL, Shajahan AN, Clarke R. Autophagy and endocrine resistance in breast cancer. Expert Rev Anticancer Ther 2011; 11: 1283-94.

25. Liu LZ, Zhou XD, Qian G, et al. AKT1 amplification regulates cisplatin resistance in human lung cancer cells through the mammalian target of rapamycin/p70S6K1 pathway. Cancer Res 2007; 67: 6325-32.

26. Spankuch B, Kurunci-Csacsko E, Kaufmann M, et al. Rational combinations of siRNAs targeting Plk1 with breast cancer drugs. Oncogene 2007; 26: 5793-807.
27. Garnett MJ, Mansfeld J, Godwin C, et al. UBE2S elongates ubiquitin chains on APC/C substrates to promote mitotic exit. Nat Cell Biol 2009; 11: 1363-9.

28. Jansen MP, Foekens JA, van Staveren IL, et al. Molecular classification of tamoxifen-resistant breast carcinomas by gene expression profiling. J Clin Oncol 2005; 23: 732-40.

29. Turner JG, Sullivan DM. CRM1-mediated nuclear export of proteins and drug resistance in cancer. Curr Med Chem 2008; 15: 2648-55.

30. Mitamura $\mathrm{T}$, Watari $\mathrm{H}$, Wang L, et al. Downregulation of miRNA-31 induces taxane resistance in ovarian cancer cells through increase of receptor tyrosine kinase MET. Oncogenesis 2013; 2: e40.

31. Devarajan E, Sahin AA, Chen JS, et al. Down-regulation of caspase 3 in breast cancer: a possible mechanism for chemoresistance. Oncogene 2002; 21: 8843-51.

32. Yamazaki T, Sasaki N, Nishi M, et al. Augmentation of drug-induced cell death by ER protein BRI3BP. Biochem Biophys Res Commun 2007; 362: 971-5.

33. Xu M, Takanashi M, Oikawa K, et al. USP15 plays an essential role for caspase- 3 activation during paclitaxelinduced apoptosis. Biochem Biophys Res Commun 2009; 388: $366-71$.

34. Quinn JE, Kennedy RD, Mullan PB, et al. BRCA1 functions as a differential modulator of chemotherapy-induced apoptosis. Cancer Res 2003; 63: 6221-8.

35. Bayraktar S, Gutierrez Barrera AM, Liu D, et al. USP-11 as a predictive and prognostic factor following neoadjuvant therapy in women with breast cancer. Cancer J 2013; 19: $10-7$.

36. Zavyalova MV, Litvyakov NV, Garbukov EY, et al. Relationship between tumor sensitivity to neoadjuvant chemotherapy and histologic pattern of primary tumor in unicentric infiltrating ductal breast carcinoma. Sib J Oncol 2008; 6: 30-4.

37. Palmieri F. The mitochondrial transporter family SLC25: identification, properties and physiopathology. Mol Aspects Med 2013; 34: 465-84. 